CERN-EP/2001-083

14 November 2001

\title{
STUDY OF $D^{\star+}$ PRODUCTION IN $\nu_{\mu}$ CHARGED CURRENT INTERACTIONS IN THE NOMAD EXPERIMENT
}

\section{The NOMAD Collaboration}

P. Astier ${ }^{1)}$ D. Autiero ${ }^{2)}$ A. Baldisseri ${ }^{3)}$ M. Baldo-Ceolin ${ }^{4)}$ M. Banner ${ }^{1)}$ G. Bassompierre $^{5)}$ K. Benslama ${ }^{6)}$ N. Besson ${ }^{3)}$ I. Bird ${ }^{2,6)}$ B. Blumenfeld ${ }^{7}$ F. Bobisut ${ }^{4)}$ J. Bouchez $^{3)}$ S. Boyd $^{8)}$ A. Bueno ${ }^{9,10)}$ S. Bunyatov ${ }^{11)}$ L. Camilleri ${ }^{2)}$ A. Cardini ${ }^{12)}$ P.W. Cattaneo ${ }^{13)}$ V. Cavasinni ${ }^{14)}$ A. Cervera-Villanueva ${ }^{2,15)}$ R. Challis ${ }^{20)}$ A. Chukanov ${ }^{11)}$ G. Collazuol ${ }^{4)}$ G. Conforto ${ }^{2,16)}$ C. Conta ${ }^{13)}$ M. Contalbrigo ${ }^{4)}$ R. Cousins ${ }^{12)}$ D. Daniels ${ }^{9)}$ H. Degaudenzi ${ }^{6)}$ T. Del Prete ${ }^{14)}$ A. De Santo ${ }^{2)}$ T. Dignan ${ }^{9)}$ L. Di Lella ${ }^{2)}$ E. do Couto e Silva ${ }^{2)}$ J. Dumarchez ${ }^{1)}$ M. Ellis ${ }^{8}$ T. Fazio ${ }^{5)}$ G.J. Feldman ${ }^{9)}$ R. Ferrari ${ }^{13)}$ D. Ferrère ${ }^{2)}$ V. Flaminio ${ }^{14)}$ M. Fraternali ${ }^{13)}$ J.-M. Gaillard ${ }^{5)}$ E. Gangler ${ }^{2,1)}$ A. Geiser ${ }^{17,2)}$ D. Geppert ${ }^{17)}$ D. Gibin ${ }^{4)}$ S. Gninenko ${ }^{2,18)}$ A. Godley ${ }^{23,8)}$ J.-J. Gomez-Cadenas ${ }^{2,15)}$ J. Gosset $^{3)}$ C. Gößling ${ }^{17)}$ M. Gouanère ${ }^{5)}$ A. Grant ${ }^{2)}$ G. Graziani ${ }^{19)}$ A. Guglielmi ${ }^{4)}$ C. Hagner ${ }^{3)}$ J. Hernando $^{15)}$ D. Hubbard ${ }^{9)}$ P. Hurst ${ }^{9)}$ N. Hyett ${ }^{20)}$ E. Iacopini ${ }^{19)}$ C. Joseph ${ }^{6)}$ F. Juget ${ }^{6)}$ N. Kent ${ }^{20)}$ M. Kirsanov ${ }^{18)}$ O. Klimov ${ }^{11)}$ J. Kokkonen ${ }^{2)}$ A. Kovzelev ${ }^{18,13)}$ A. Krasnoperov ${ }^{5,11)}$ D. Kustov ${ }^{11)}$ V. Kuznetsov ${ }^{11,2)}$ S. Lacaprara ${ }^{4)}$ C. Lachaud ${ }^{1)}$ B. Lakić( ${ }^{21)}$ A. Lanza ${ }^{13)}$ L. La Rotonda ${ }^{22)}$ M. Laveder ${ }^{4)}$ A. Letessier-Selvon ${ }^{1)}$ J.-M. Levy ${ }^{1)}$ L. Linssen $^{2)}$ A. Ljubičićc ${ }^{21)}$ J. Long $^{7)}$ A. Lupi ${ }^{19)}$ A. Marchionni ${ }^{19)}$ F. Martelli ${ }^{16)}$ X. Méchain ${ }^{3)}$ J.-P. Mendiburu ${ }^{5)}$ J.-P. Meyer ${ }^{3)}$ M. Mezzetto ${ }^{4)}$ S.R. Mishra ${ }^{9,23)}$

G.F. Moorhead ${ }^{20)}$ D. Naumov ${ }^{11)}$ P. Nédélec ${ }^{5)}$ Yu. Nefedov ${ }^{11)}$ C. Nguyen-Mau ${ }^{6)}$ D. Orestano ${ }^{24)}$ F. Pastore ${ }^{24)}$ L.S. Peak ${ }^{8)}$ E. Pennacchio ${ }^{16)}$ H. Pessard ${ }^{5)}$ R. Petti2 ${ }^{2,13)}$ A. Placci' ${ }^{2)}$ G. Polesello ${ }^{13)}$ D. Pollmann ${ }^{17)}$ A. Polyarush ${ }^{18)}$ B. Popov ${ }^{11,1)}$ C. Poulsen ${ }^{20)}$ J. Rico ${ }^{10)}$ P. Riemann ${ }^{17)}$ C. Roda ${ }^{2,14)}$ A. Rubbia ${ }^{2,10)}$ F. Salvatore ${ }^{13)}$ K. Schahmaneche ${ }^{1)}$ B. Schmidt ${ }^{17,2)}$ T. Schmidt ${ }^{17)}$ M. Sevior ${ }^{20)}$ D. Sillou ${ }^{5)}$ F.J.P. Soler ${ }^{2,8)}$ G. Sozzi ${ }^{6)}$ D. Steele ${ }^{7,6)}$ U. Stiegler ${ }^{2)}$ M. Stipčević ${ }^{21)}$ Th. Stolarczyk ${ }^{3)}$ M. Tareb-Reyes ${ }^{6)}$ G.N. Taylor ${ }^{20)}$ V. Tereshchenko ${ }^{11)}$ A. Toropin ${ }^{18)}$ A.-M. Touchard ${ }^{1)}$ S.N. Tovey ${ }^{2,20)}$ M.-T. Tran ${ }^{6)}$ E. Tsesmelis ${ }^{2)}$ J. Ulrichs ${ }^{8)}$ L. Vacavant ${ }^{6)}$ M. Valdata-Nappi ${ }^{22, *)}$ V. Valuev ${ }^{11,12)}$ F. Vannucci ${ }^{1)}$ K.E. Varvell ${ }^{8)}$ M. Veltri ${ }^{16)}$ V. Vercesi ${ }^{13)}$ G. Vidal-Sitjes ${ }^{2)}$ J.-M. Vieira ${ }^{6)}$ T. Vinogradova ${ }^{12)}$ F.V. Weber ${ }^{9,2)}$ T. Weisse ${ }^{17)}$ F.F. Wilson ${ }^{2)}$ L.J. Winton ${ }^{20)}$ B.D. Yabsley $^{8)}$ H. Zaccone ${ }^{3)}$ K. Zuber ${ }^{17)}$ P. Zuccon ${ }^{4)}$

\begin{abstract}
A search was made among $\nu_{\mu}$ charged current events collected in the NOMAD experiment for the reaction:

$$
\begin{aligned}
\hookrightarrow & \mathrm{D}^{0}+\pi^{+} \\
& \hookrightarrow \mathrm{K}^{-}+\pi^{+}
\end{aligned}
$$$$
\nu_{\mu}+N \rightarrow \mu^{-}+D^{\star+}+\text { hadrons }
$$

A high purity $D^{\star+}$ sample composed of 35 events was extracted. The $D^{\star+}$ yield in $\nu_{\mu}$ charged current interactions was measured to be $T=(0.79 \pm 0.17$ (stat. $) \pm$ 0.10 (syst.) $) \%$. The mean fraction of the hadronic jet energy taken by the $D^{\star+}$ is $0.67 \pm 0.02$ (stat) \pm 0.02 (syst.). The distributions of the fragmentation variables $z$, $P_{T}^{2}$ and $x_{F}$ for $D^{\star+}$ are also presented.
\end{abstract}

Preprint submitted for publication in Phys. Lett.B. 


\footnotetext{
1) LPNHE, Univ. of Paris VI and VII, Paris, France

2) CERN, Geneva, Switzerland

3) DAPNIA, CEA Saclay, France

4) Univ. of Padova and INFN, Padova, Italy

5) LAPP, Annecy, France

6) University of Lausanne, Lausanne, Switzerland

7) Johns Hopkins Univ., Baltimore, MD, USA

8) Univ. of Sydney, Sydney, Australia

9) Harvard Univ., Cambridge, MA, USA

10) ETH Zürich, Zürich, Switzerland

11) JINR, Dubna, Russia

12) UCLA, Los Angeles, CA, USA

13) Univ. of Pavia and INFN, Pavia, Italy

14) Univ. of Pisa and INFN, Pisa, Italy

15) IFIC, Valencia, Spain

16) Univ. of Urbino, Urbino, and INFN Florence, Italy

17) Dortmund Univ., Dortmund, Germany

18) Inst. Nucl. Research, INR Moscow, Russia

19) Univ. of Florence and INFN, Florence, Italy

20) University of Melbourne, Melbourne, Australia

${ }^{21)}$ Rudjer Bošković Institute, Zagreb, Croatia

${ }^{22)}$ Univ. of Calabria and INFN, Cosenza, Italy

23) Univ. of South Carolina, Columbia, SC, USA

24) Roma Tre University and INFN, Rome, Italy

*) Now at Univ. of Perugia and INFN, Perugia, Italy
} 


\section{INTRODUCTION}

The main goal of the NOMAD experiment is the study of the neutrino oscillation $\nu_{\mu} \rightarrow \nu_{\tau}$ using the CERN SPS wide-band neutrino beam. The search uses kinematic criteria to identify $\nu_{\tau}$ charged current interactions $\left(\nu_{\tau} \mathrm{CC}\right)$ [1]. A set of drift chambers in a magnetic field $(0.4 T)$ was used to reconstruct charged particle momenta [2]. The apparatus has been extensively described in [3]. The oscillation search in NOMAD requires a precise measurement of all visible final-state particles and an efficient event reconstruction. The detector is optimized to have good energy and momentum resolution [3]. The sample of $1.3 \times 10^{6} \nu_{\mu}$ charged current interactions $\left(\nu_{\mu} \mathrm{CC}\right)$, collected during four years (19951998), allows the measurement of open charm production. In this paper, we present the study of $D^{\star+}$ production using its hadronic decays. This choice allows the measurement of all the $D^{\star+}$ decay products lending some insight into the charm production mechanism. Using the extracted sample of $D^{\star+}$, we also report on the $z, x_{F}$ and $P_{T}^{2}$ dependence of $D^{\star+}$ production.

\section{CHARM PRODUCTION}

Charm production in neutrino charged current interactions has first been studied using opposite sign dimuons produced in charged current interactions at a rate of about $0.6 \%$ for neutrino energies below $150 \mathrm{GeV}$ [4]. However, these results have been obtained using massive detectors which prevent the direct observation of the charmed particles. Charm production was also studied in dilepton $\left(\mu^{-} e^{+}\right)$production [5] with $P_{e^{+}}>300$ $\mathrm{MeV} / \mathrm{c}$ and a rate of $(0.42 \pm 0.06) \%$. Only a few bubble chamber and emulsion experiments [6][7] have actually reconstructed the charmed particle decays.

NOMAD has also published a study of charm through dimuon events produced in a massive calorimeter preceding the standard NOMAD target [9]. This study was based on $30 \%$ of the available data, the full data sample being currently analyzed. The analysis presented in this paper aims at identifying fully reconstructed charm events in order to separate the various contributions to the overall charm production rate measured through dimuon events. In the NOMAD detector the space resolution is not sufficient for a reconstruction of the decay vertex of the charmed meson, separating it from the primary interaction point. One therefore must rely on the measurement of the momenta of all the produced hadrons and on kinematical methods for the selection of the $D^{\star+}$ within the hadronic jet. We have in particular chosen the following purely hadronic channel, for which all the decay products are measured in the detector:

$$
\begin{array}{rlrl}
D^{\star+} \rightarrow \mathrm{D}^{0}+\pi^{+} & \text {B.R } & =(67.7 \pm 0.5) \% \\
\hookrightarrow \mathrm{K}^{-}+\pi^{+} & \text {B.R }=(3.83 \pm 0.09) \%
\end{array}
$$

The $D^{\star+}$ yield in $\nu_{\mu} \mathrm{CC}$ interactions has been measured previously in the BEBC experiments to be $T=(1.22 \pm 0.25) \%$ [6]. With this value, we expect an observable rate of $(3.16 \pm 0.75) \times 10^{-4} D^{\star+}$ per $\nu_{\mu}$ CC events.

The mean neutrino energy of our $\nu_{\mu} \mathrm{CC}$ events was $45.3 \mathrm{GeV}$. In order to estimate the background, we have used a Monte Carlo sample of about $3.3 \times 10^{6} \nu_{\mu}$ CC events. In addition, we have generated a sample of $13 \times 10^{3} D^{\star+}$ events in the decay channel studied. Our simulation program is based on a version of LEPTO 6.1 [10] and JETSET 7.4 [11] with the $Q^{2}$ and $W^{2}$ cutoff parameters removed. To define the parton content of the nucleon for the cross-section calculation we have used the GRV-HO parametrization [12] of the 
parton density functions available in PDFLIB [13]. The nucleon Fermi motion distribution of reference [14], truncated at $1 \mathrm{GeV} / \mathrm{c}$ was also used. A full detector simulation based on GEANT[15] was performed.

\section{CANDIDATE SELECTION}

A sample of $\nu_{\mu} \mathrm{CC}$ events was selected requiring at least one primary negative muon identified in the muon chambers and with a momentum greater than $3 \mathrm{GeV} / \mathrm{c}$. The tracks used to reconstruct the $D^{\star+}$ candidate were all the primary tracks except for those identified as electrons in the Transition Radiation Detector [16] or as muons in the muon chambers. The selected non-leptonic tracks were combined to reconstruct the $D^{0}$ candidate, building the invariant mass $m_{(+-)}$with a positive and a negative track to which we have assigned a $\pi$ mass and a $K$ mass respectively. The $D^{\star+}$ candidate was obtained by adding a low momentum $(<4 \mathrm{GeV} / \mathrm{c})$ positive track, assigning it a $\pi^{+}$mass, to the previous ones to build the invariant mass $m_{(+-)+}$. This last track corresponds to the low energy (slow) pion coming from the $D^{\star+}$ decay and will be referred to as $\pi_{s}$ subsequently. The combinatorial background was reduced using four cuts exploiting the kinematics of $D^{\star+}$ and $D^{0}$ decays:

$-P_{\pi_{s}}^{T}: \pi_{s}$ transverse momentum with respect to the $D^{\star+}$ momentum direction.

$P_{\pi_{s}}^{T}<0.06 \mathrm{GeV} / \mathrm{c}$

$-\theta_{\pi_{s} \mathrm{D}^{0}}$ : laboratory angle between $\pi_{s}$ and $\mathrm{D}^{0}$ :

$\cos \theta_{\pi_{s} \mathrm{D}^{0}}>0.996$

$-\theta_{\pi \mathrm{K}}$ : laboratory angle between $\pi$ and $\mathrm{K}$ from the $\mathrm{D}^{0}$ decay: $\cos \theta_{\pi \mathrm{K}}>0.7$

$-\theta_{\mathrm{KD}^{0}}$ : laboratory angle between $\mathrm{K}$ and $\mathrm{D}^{0}$ :

$\cos \theta_{\mathrm{KD}^{0}}>0.95$

The values of the last two cuts have been optimized using the simulation.

With these cuts, we find a single $D^{\star+}$ candidate in $96 \%$ of the surviving data and $\mathrm{MC}$ events. For the remaining events, in which there are several candidates, we have kept the track combination that minimizes the quantity $|\Delta m-0.1454| \mathrm{GeV} / \mathrm{c}^{2}$ where $\Delta m=m_{(+-)+}-m_{(+-)}$and $0.1454 \mathrm{GeV} / \mathrm{c}^{2}$ is the mass difference $m_{D^{\star+}}-m_{D^{0}}$ [17]. At this stage the signal to background ratio in the MC sample was $\sim \frac{1}{38}$. To improve upon this ratio we have used a neural network method which allowed the selection of samples with a signal purity as high as $90 \%$.

\section{NEURAL NETWORK METHOD}

We have used the JETNET package [18], choosing a "feed-forward" neural network with a "back-propagation" learning. For this treatment we have kept the events which satisfy:

$$
\Delta m=m_{(+-)+}-m_{(+-)}<0.2 \mathrm{GeV} / \mathrm{c}^{2}
$$

The training sample contained $2060 \mathrm{MC}$ signal events taken from the simulated signal sample and 2117 background events taken from the $\nu_{\mu} \mathrm{CC}$ simulated sample. Different network structures have been tested with a single hidden layer. The last layer was always composed of 2 outputs. However, as the results did not show any difference between these two outputs, we used only the first one. We tuned the number of nodes in the hidden layer and the number of variables at the input layer. We kept the structure which gave the best 
signal selection efficiency for a given background rejection level. The network was trained during a large number of epochs (30000). During the training, we have tested the structure every 100 epochs. The test consisted of minimizing the background contamination in the final selected sample. The contamination was computed for events in the range $\mid m_{(+-)+}$ 2.01| $<0.05 \mathrm{GeV} / \mathrm{c}^{2}$. We have kept the weight values which gave the best signal selection efficiency. The network training was performed by requiring a given output value as shown in Figure 1. The test procedure was done with events not used in the training: 2891 events from the simulated signal sample were used to compute the signal efficiency and 8447 events (8083 background events $+364 D^{\star+}$ events) to estimate the background contamination. Eight variables were finally used as inputs:

1. $\cos \theta^{\star}$ : cosine of the angle between $D^{0}$ and $\pi^{+}$momenta in the $D^{0}$ center of mass

2. $P_{\pi}^{T}$ : transverse pion momentum with respect to $P_{\mathrm{D}^{0}}$ direction from the decay

$\mathrm{D}^{0} \rightarrow \mathrm{K}^{-}+\pi^{+}$in the laboratory frame

3. $\Delta m=\left|m_{(+-)+}-m_{(+-)}\right|$

4. $P_{\pi_{s}}$ : pion momentum from $D^{\star+}$ decay in the laboratory

5. $P_{\pi_{s}}^{\star}$ : pion momentum from $D^{\star+}$ in $D^{\star+}$ center of mass

6. $\theta_{P_{\text {had }} \pi_{s}}$ : angle between hadronic jet momentum and pion momentum from the decay $D^{\star+} \rightarrow \mathrm{D}^{0}+\pi^{+}$

7. $\theta_{P_{\text {had. }} D^{\star+}}$ : angle between the direction of the hadronic jet momentum and the $D^{\star+}$ momentum

8. $\theta_{P_{\text {had. }}}$ : angle between the direction of the hadronic jet momentum direction and the neutrino direction

The structure which provides an optimal signal selection efficiency for a $10 \%$ background contamination has 8 inputs, 12 nodes on the hidden layer and 2 outputs. The values of the output, $K$, given by the network for the simulated test sample and for the data are shown in Figure 2.

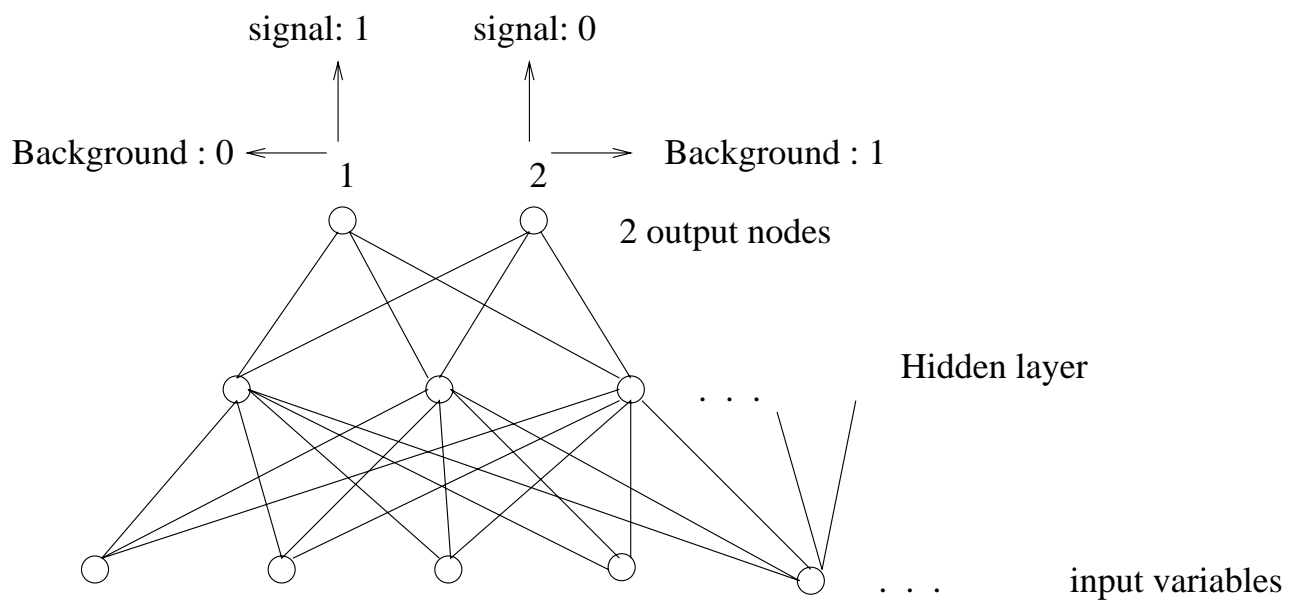

Figure 1: Neural network used in this selection. Only output 1 is used in the signal selection.

\section{SIGNAL SELECTION}

With the structure (8-12-2) chosen here, we computed the number of signal events in the data applying two selection criteria. We required $K$ larger than 0.8735 , to define a 
sample containing a background contamination smaller than $10 \%$ (Fig. 2) resulting in a signal efficiency of $(13.1 \pm 0.4) \%$. The resulting $m_{(+-)+}$mass distributions are shown in Figure 3 for MC and data. A clear peak is seen at the $D^{\star+}$ mass, amounting to 47 events in the mass interval $\left|m_{(+-)+}-2.01\right|<0.05 \mathrm{GeV} / \mathrm{c}^{2}$. Interpolating the background from outside the signal mass interval gives a total number of $(35 \pm 7.2) D^{\star+}$ events.

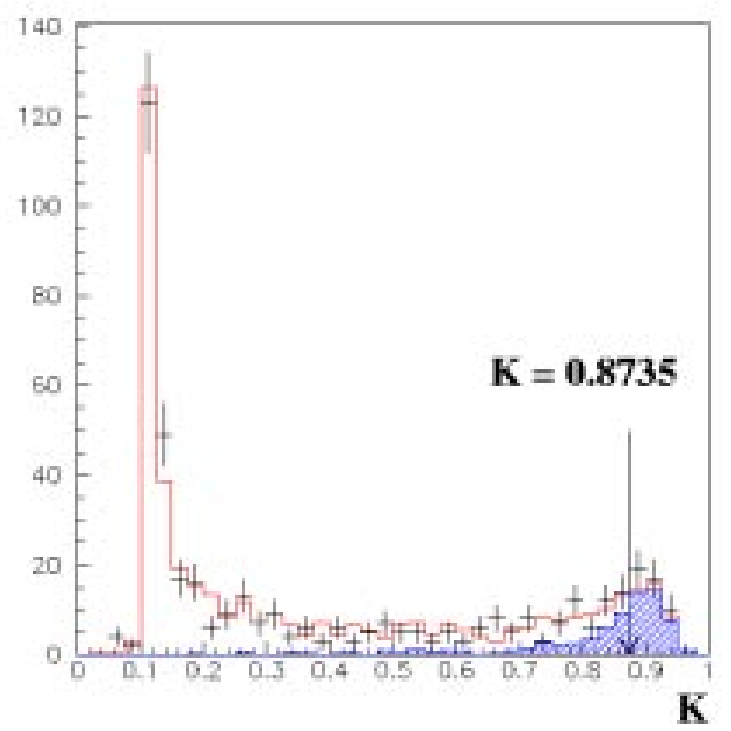

Figure 2: Comparison between the output value $\mathrm{K}$ given by the network for data (points with error bars) and MC test sample (histogram) for events falling in the window $\left|m_{(+-)+}-2.01\right|<0.05 \mathrm{GeV} / \mathrm{c}^{2}$. The MC signal component is shaded. Selecting events with $K>0.8735$ results in a signal sample with a $90 \%$ purity. The MC distributions are normalised to the data.

We then reduced the threshold on $K$ to 0.6 to increase the signal efficiency to more than $30 \%$. For this threshold, the remaining background was large and the Monte Carlo simulation showed that the background was purely combinatorial. We used the $m_{(+-)+}$ reconstructed mass distribution to estimate this background using three methods based on the MC sample of $\nu_{\mu} \mathrm{CC}$ events (Fig. 4):

1. For each MC event, we know whether it is a $D^{\star+}$ event or a background event. The shape of the background distribution can be deduced from this sample.

2. We apply the same selections and cuts as in section 3 to $\mathrm{MC}$ events but instead of reconstructing $m_{(+-)+}$, we select $m_{(-+)-}$. Since $D^{\star-}$, which would require the production of $\bar{c}$ quark, which are produced at a negligeable rate in $\nu_{\mu} C C$ events, we are left with only background.

3. With each mass combination $m_{(+-)}$we have associated a positive track taken from another $\mathrm{MC}$ event and required that this combination passes the selection and the cuts used in section 3.

The estimations of the background 2 and 3 above were verified with the data sample which was 3 times less copious than the MC one .

Each background estimation was subtracted from the data after normalisation in the region $m_{(+-)+}<1.9 \mathrm{GeV} / \mathrm{c}^{2}$ and $m_{(+-)+}>2.1 \mathrm{GeV} / \mathrm{c}^{2}$ (Fig. 4). The number of signal events, averaged over the three background subtraction methods is: $N_{\text {evt }}=85.7 \pm 18.5$. 

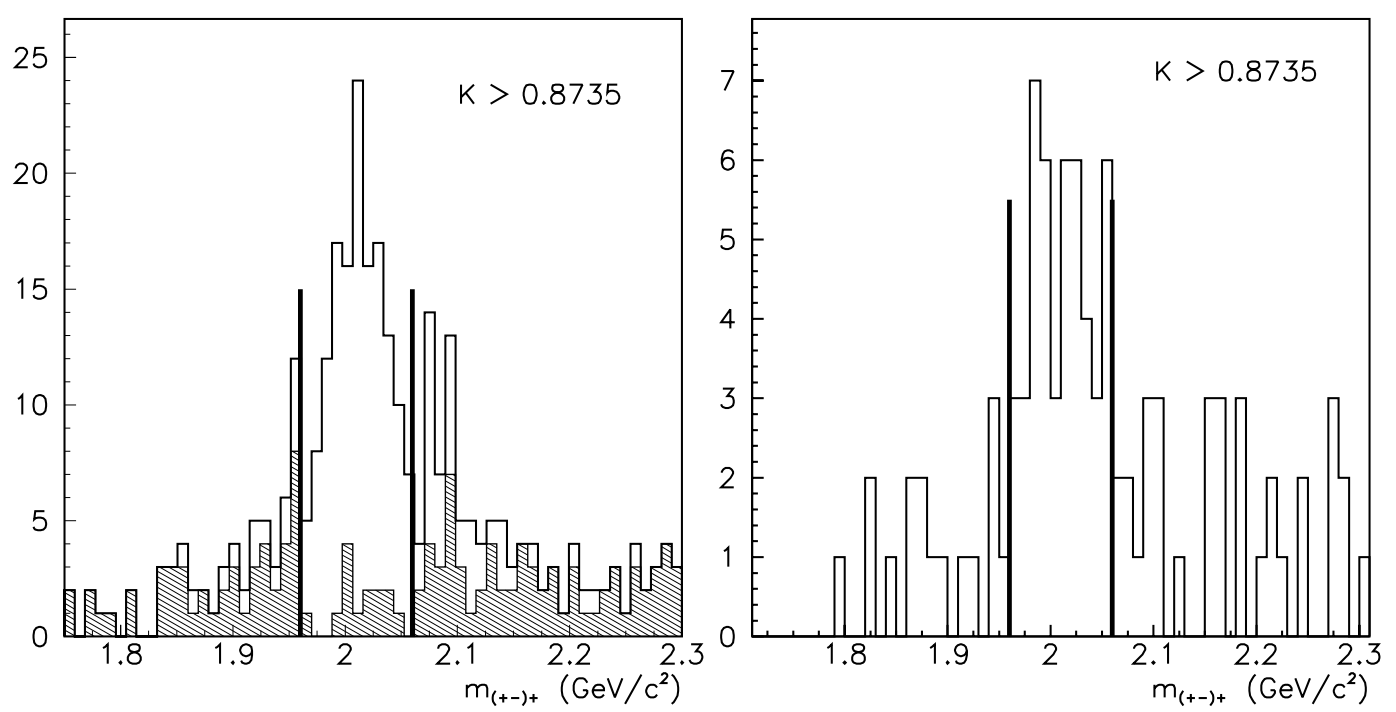

Figure 3: The $m_{(+-)+}$distribution for MC (left) and for data (right) for events with $K$ larger than 0.8735. The vertical lines show the window $\left(\left|m_{(+-)+}-2.01\right|<0.05 \mathrm{GeV} / \mathrm{c}^{2}\right)$ used for the signal definition. The shaded area on the left plot is the background contribution.

The corresponding signal efficiency is $(33.8 \pm 0.5) \%$.

Two alternative analysis methods were also used, one based on a standard sequential cuts method and the other based on a likelihood function. The results of the three analyses are listed in Table 1 which gives the number of identified $D^{\star+}$, the efficiencies and the total efficiency corrected number of $D^{\star+}$ for the given decay mode. The three methods yield results which are in very good agreement with each other. Note that the cleanest sample with the highest efficiency is obtained from the neural network analysis. This will therefore be used for the fragmentation study.

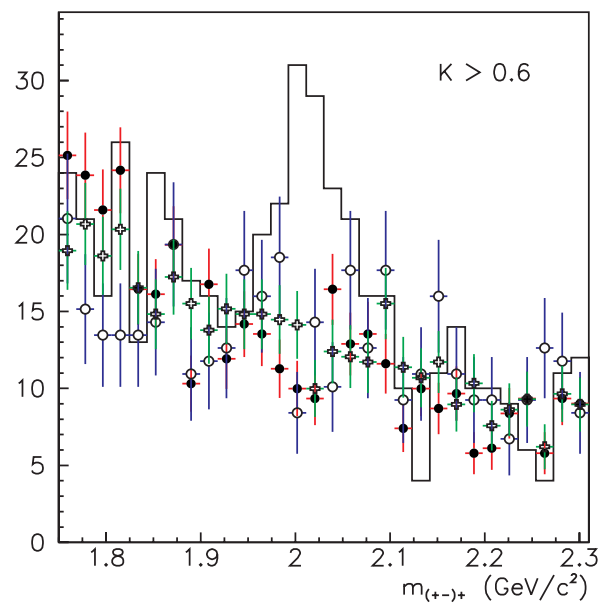

Figure 4: Data $m_{(+-)+}$distribution for events with $K$ larger than 0.6 with the three normalized background estimations: MC (full circles), inverted charged combination (open circles) and with a third track taken from another event (open crosses). 


\begin{tabular}{|c||c|c|c|}
\hline & $\begin{array}{c}\text { Eff.(\%) } \\
\pm \text { stat. }\end{array}$ & $\begin{array}{c}N_{\text {evt }} \\
\pm \text { stat. }\end{array}$ & $\begin{array}{c}\text { Total number } \\
\text { of signal events } \\
\text { produced } \\
\pm \text { stat. } \pm \text { syst. }\end{array}$ \\
\hline $\begin{array}{c}\text { Neural } \\
\text { network } \\
\text { (high purity) }\end{array}$ & $\begin{array}{c}13.1 \\
\pm 0.4\end{array}$ & $\begin{array}{c}35 \\
\pm 7.2\end{array}$ & $\mathbf{2 6 7} \pm \mathbf{5 5} \pm \mathbf{2 6}$ \\
\hline $\begin{array}{c}\text { Neural } \\
\text { network } \\
\text { (eff. } \simeq 30 \%)\end{array}$ & $\begin{array}{c}33.8 \\
\pm 0.5\end{array}$ & $\begin{array}{c}85.7 \\
\pm 18.5\end{array}$ & $\mathbf{2 5 4} \pm \mathbf{5 5} \pm \mathbf{2 9}$ \\
\hline \hline $\begin{array}{c}\text { Sequential } \\
\text { cuts } \\
\text { (eff. } \simeq 30 \%)\end{array}$ & $\begin{array}{c} \pm 0.6 \\
\pm 0.4\end{array}$ & $\begin{array}{c}84.4 \\
\pm 17.6\end{array}$ & $\mathbf{2 7 6} \pm \mathbf{5 8} \pm \mathbf{3 2}$ \\
\hline $\begin{array}{c}\text { Likelihood } \\
\text { ratio } \\
\text { (high purity) }\end{array}$ & $\begin{array}{c}9.7 \\
\pm 0.4\end{array}$ & $\begin{array}{c} \pm 0 \\
\pm 5\end{array}$ & $\mathbf{2 7 4} \pm \mathbf{4 8} \pm \mathbf{3 4}$ \\
\hline $\begin{array}{c}\text { Likelihood } \\
\text { ratio } \\
\text { (eff. } \simeq 30 \% \text { ) }\end{array}$ & $\begin{array}{c} \pm 3.3 \\
\pm 0.6\end{array}$ & $\begin{array}{c} \pm 1.2 \\
\pm 18.6\end{array}$ & $\mathbf{2 7 4} \pm \mathbf{5 6} \pm \mathbf{3 2}$ \\
\hline
\end{tabular}

Table 1: Number of signal events obtained using the selection method described in the text, and using two alternative methods (sequential cuts and likelihood ratio). The total number of signal events (last column) has been obtained by dividing $N_{\text {evt }}$ (third column) by the efficiency (second column).

\section{FRAGMENTATION STUDY}

The fragmentation of charmed mesons can be described by three variables: the fraction $z$ of the total hadronic jet energy carried by the meson, the Feynman $x_{F}$ variable defined as the ratio of the meson longitudinal momentum in the hadronic rest system to the maximum possible for this momentum, and the transverse momentum $P_{T}$ of the charmed meson with respect to the direction of the hadronic system.

The $z, x_{F}$ and $P_{T}^{2}$ distributions of the 47 events selected with the neural network method with high purity were obtained. From these we subtracted the contribution of the 12 background events as determined from events in the invariant side band regions $\left(1.80 \mathrm{GeV} / \mathrm{c}^{2}<m_{(+-)+}<1.96 \mathrm{GeV} / \mathrm{c}^{2}\right)$ and $\left(2.06 \mathrm{GeV} / \mathrm{c}^{2}<m_{(+-)+}<2.22 \mathrm{GeV} / \mathrm{c}^{2}\right)$. The resulting distributions have been corrected for the detector efficiency and renormalized to the number of signal events (35). The $z$ distribution can be parametrized using the Collins-Spiller [19] or the Peterson [20] functions, $D_{c}(z)$ and $D_{p}(z)$ respectively:

$$
\begin{aligned}
& D_{c}(z)=N\left(\frac{1-z}{z}+\frac{\epsilon_{c}(2-z)}{1-z}\right)\left(1+z^{2}\right)\left(1-\frac{1}{z}-\frac{\epsilon_{c}}{1-z}\right)^{-2} \\
& D_{p}(z)=\frac{N}{z\left(1-1 / z-\epsilon_{p} /(1-z)\right)^{2}}
\end{aligned}
$$

$N$ is a normalisation factor and $\epsilon_{c}$ and $\epsilon_{p}$ are free parameters to be determined by a fit to the data. 
The $P_{T}^{2}$ distribution can be parametrized as: $N \exp \left[-B\left(m^{2}+P_{T}^{2}\right)^{\frac{1}{2}}\right]$ [7], where $\mathrm{N}$ is a normalisation factor and $B$ and $m$ are free parameters to be determined by a fit to the data or set to given values.

The mean values of $x_{F}$ and $z$ that we find, $\left\langle x_{F}\right\rangle=0.47$ and $\langle z\rangle=0.67$, clearly show that the $D^{\star+}$ is produced forward with respect to the jet and carries a large fraction of the jet energy. The results of the fragmentation study are given in Table 2 and in Figures 5 and 6.
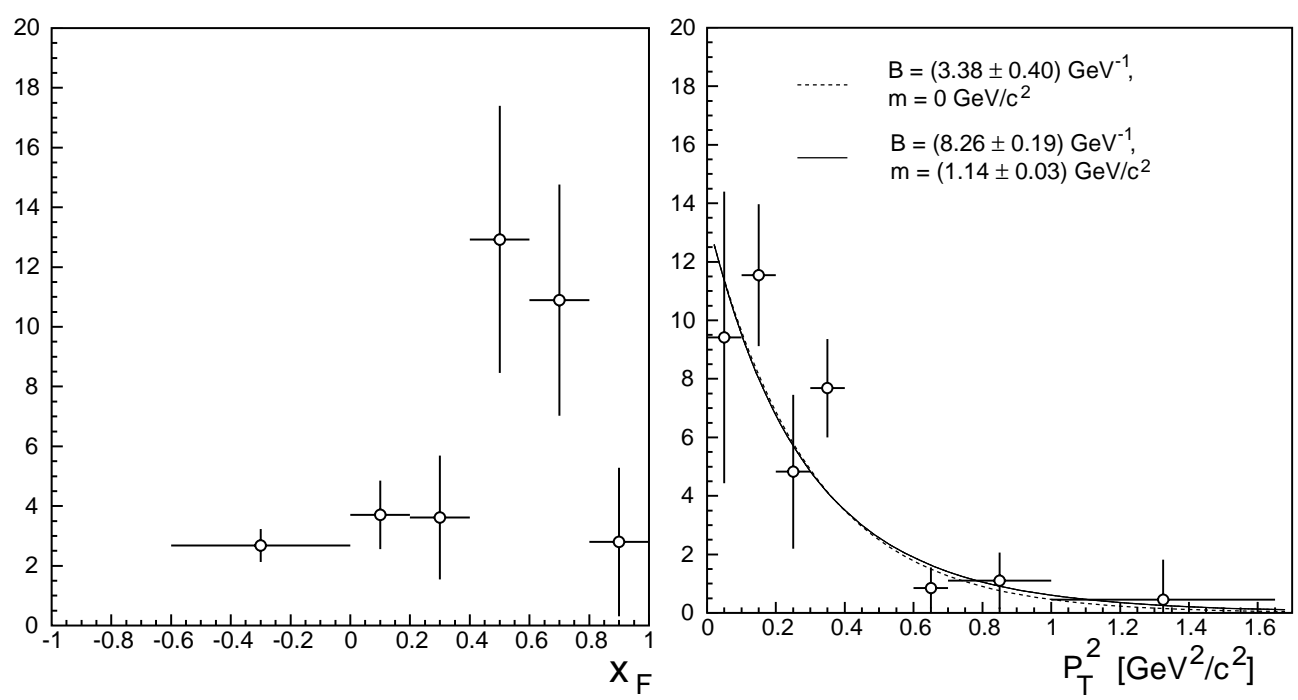

Figure 5: $x_{F}$ distribution (left) and $P_{T}^{2}$ distribution (right) for the events selected by the neural network method with a high purity.

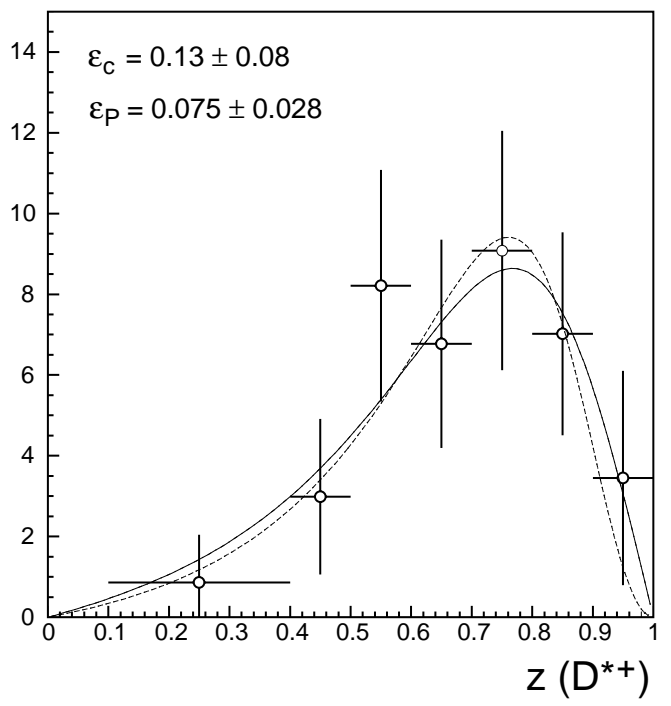

Figure 6: $z$ distribution for the events selected by the neural network method with a high purity. The fits obtained using the parametrisation given in the text and the values of $\epsilon_{c}$ and $\epsilon_{p}$ in Table 2 are also shown. 


\begin{tabular}{|c|c|c|}
\hline Variables & $\begin{array}{l}\text { Mean value } \\
\pm \text { stat. } \pm \text { syst. }\end{array}$ & $\begin{array}{c}\text { Fit value } \\
\pm \text { stat. } \pm \text { syst. }\end{array}$ \\
\hline \multirow[t]{3}{*}{$z$} & $\begin{array}{c}0.67 \\
\pm 0.02 \pm 0.02\end{array}$ & \\
\hline & & $\begin{array}{c}\epsilon_{p}=0.075 \\
\pm 0.028 \pm 0.036\end{array}$ \\
\hline & & $\begin{array}{c}\epsilon_{c}=0.13 \\
\pm 0.08 \pm 0.11\end{array}$ \\
\hline$x_{F}$ & $\begin{array}{c}0.47 \\
\pm 0.05(\text { stat. })\end{array}$ & \\
\hline $\begin{array}{c}P_{T} \\
(\mathrm{GeV} / \mathrm{c})\end{array}$ & $\begin{array}{c}0.49 \\
\pm 0.05(\text { stat. })\end{array}$ & \\
\hline $\begin{array}{c}P_{T}{ }^{2} \\
(\mathrm{GeV} / \mathrm{c})^{2}\end{array}$ & $\begin{array}{c}0.23 \\
\pm 0.06(\text { stat. })\end{array}$ & \\
\hline & & $\begin{array}{c}\mathrm{B}=3.38 \pm 0.40(\text { stat. }) \\
m=0 .\end{array}$ \\
\hline & & $\begin{array}{l}\mathrm{B}=8.26 \pm 0.19 \text { (stat. }) \\
m=1.14 \pm 0.03(\text { stat. })\end{array}$ \\
\hline
\end{tabular}

Table 2: Results of the $D^{\star+}$ fragmentation study. The third column gives the values of the fitted parameters for the $z$ and $P_{T}{ }^{2}$ distributions. For this latter case two fits were performed using the parametrization $N \exp \left[-B\left(m^{2}+P_{T}^{2}\right)^{\frac{1}{2}}\right]$, one with the $m$ parameter set to zero and the other with $m$ as a free parameter.

\section{SYSTEMATIC ERRORS}

Systematic errors on the number of $D^{\star+}$ events, as well as on the parameters of the $x_{F}, P_{T}^{2}$ and $z$ distributions arise from the initial cuts that we have applied in order to suppress the background, as well as from the chosen neural network method. The effect on the final result of the experimental uncertainties on the variables used for selecting the signal, was studied by changing each variable in turn by a quantity equal to its uncertainty, and repeating the analysis. The uncertainty on the background level under the " $90 \%$ purity" peak (see section 5) was also included in the systematic errors. The systematic error was obtained by adding each contribution in quadrature. It is worth noting that in this estimation, the selection efficiency does vary slightly and that this variation is accounted for. For the neural network method, to determine the bias introduced by the choice of the training sample, we have built two new training samples out of the Monte Carlo events and repeated the whole selection procedure. Here again, the systematic error was estimated using the deviation from the standard value.

Fragmentation The systematic error on the fraction of energy carried by the $D^{\star+}$ over the total hadronic jet energy has two origins. The first one comes from the errors on the energy of the $D^{\star+}$ meson and the second one from the estimation of the total hadronic jet energy. Monte Carlo studies showed that the total hadronic jet energy can be underestimated by at most $10 \%$. Combining these two gives an error on $\langle z\rangle$ of 0.01 . In addition, the procedure described above to study the systematics using the neural network also showed that the mean value of $z$ has a systematic uncertainty of 0.02 . The total systematic error on $\langle z\rangle$, 
therefore, is 0.02 . The same procedure has been used to compute the systematic error on $\epsilon_{p}$ and $\epsilon_{c}$. The results of the fragmentation study are summarised in Table 2.

\section{DISCUSSION}

The total $D^{\star+}$ yield in $\nu_{\mu}$ CC events, $T$, computed for the selection using the neural network method for the high purity selection and the branching ratios quoted earlier, is found to be :

$$
T=(0.79 \pm 0.17(\text { stat. }) \pm 0.10(\text { syst. })) \%
$$

The BEBC experiments [6], which used a similar neutrino beam to NOMAD, have reported $D^{\star+}$ production in the hadronic channel [6]. They have obtained a $D^{\star+}$ yield of: $T=(1.22 \pm 0.25) \%$ and a mean value: $\langle z\rangle=0.59 \pm 0.03$ (stat) \pm 0.08 (syst.). These are compatible with our values. Note that the total charm yield in neutrino interactions at these energies as measured by dimuon experiments [4] is about 6\%. The E531 experiment, with a nuclear emulsion target and a neutrino beam with energy similar to the CERN SPS has also studied charm fragmentation. In their publications of 1983 [7], they report 23 charmed events, of which 15 were D mesons, and gave the following mean values for $z$ and $P_{T}:\langle z\rangle=0.59 \pm 0.04$ and $\left\langle P_{T}\right\rangle=0.64 \pm 0.16 \mathrm{GeV} / \mathrm{c}$. In two additional papers [8], the events sample was increased to 122 charmed events, of which 104 were D mesons. Fitting the $z$ distribution of the mesons they obtained $\epsilon_{p}=0.076 \pm 0.014$. Fitting the $P_{T}{ }^{2}$ distribution, with $\exp \left[-B\left(m^{2}+P_{T}^{2}\right)^{\frac{1}{2}}\right]$ they obtained $B=3.1 \mathrm{GeV}^{-1}$ assuming $m=0$ and $B=6 \mathrm{GeV}^{-1}$ assuming $m=1.3 \mathrm{GeV} / \mathrm{c}^{2}$. Their $x_{F}$ distribution is also similar to ours, as all their $D$ mesons have values of $x_{F}$ greater than -0.2 . 


\section{SUMMARY}

The production of $D^{\star+}$ in $\nu_{\mu} \mathrm{CC}$ interactions was observed through the following decay chain:

$$
\begin{aligned}
D^{\star+} \rightarrow & \mathrm{D}^{0}+\pi^{+} \\
& \hookrightarrow \mathrm{K}^{-}+\pi^{+}
\end{aligned}
$$

The total yield of $D^{\star+}$ was measured to be $(0.79 \pm 0.17$ (stat $) \pm 0.10($ syst. $\left.)\right) \%$.

With the selected $D^{\star+}$ events a study of fragmentation variables was performed. The mean value of $z$, the fraction of energy carried by the meson over the total hadronic jet energy is $\langle z\rangle=0.67 \pm 0.02$ (stat.) \pm 0.02 (syst.) The parameters of the Collins-Spiller and Peterson fragmentation functions have been obtained from a fit to the $z$ distribution: $\epsilon_{c}=$ $0.13 \pm 0.08$ (stat.) \pm 0.11 (syst.) and $\epsilon_{p}=0.075 \pm 0.028$ (stat.) \pm 0.036 (syst.). The $x_{F}$ and $P_{T}{ }^{2}$ distributions have also been extracted and the mean values are: $\left\langle x_{F}\right\rangle=0.47 \pm 0.05$ (stat.) and $\left\langle P_{T}{ }^{2}\right\rangle=0.23 \pm 0.06$ (stat.) $\mathrm{GeV}^{2} / \mathrm{c}^{2}$.

\section{Acknowledgements}

We gratefully acknowledge the CERN SPS accelerator and beam-line staff for the magnificent performance of the neutrino beam. We also thank the technical and secretarial staff of the collaborating institutes. The experiment was supported by the following funding agencies: Australian Research Council (ARC) and Department of Industry, Science, and Resources (DISR), Australia; Institut National de Physique Nucléaire et Physique des Particules (IN2P3), Commissariat à l'Energie Atomique (CEA), France; Bundesministerium für Bildung und Forschung (BMBF, contract 05 6DO52), Germany; Istituto Nazionale di Fisica Nucleare (INFN), Italy; Joint Institut for Nuclear Research and Institut for Nuclear Research of the Russian Academy of Sciences, Russia; Fonds National Suisse de la Recherche Scientifique, Switzerland; Department of Energy, National Science Foundation (grant PHY-9526278), the Sloan and the Cottrell Foundations, USA. 


\section{References}

[1] J. Altegoer et al., (NOMAD Collaboration), Phys.Lett.B 431 (1998) 219

P. Astier et al., (NOMAD Collaboration), Phys. Lett. B 453 (1999) 169

P. Astier et al., (NOMAD Collaboration), Phys. Lett. B 471 (2000) 406

P. Astier et al., (NOMAD Collaboration), Nucl. Phys. B 611 (2001) 3

[2] M. Anfreville et al., hep-ex/0104012, to be published in Nucl. Inst.and Meth.

[3] J. Altegoer et al., (NOMAD Collaboration), Nucl. Inst. Meth. A 404 (1998) 96

[4] H. Abramowicz et al., (CDHS collaboration) Z. Phys. C15 (1982) 19

A. Bazarko et al., (CCFR colllaboration), Z. Phys. C65 (1995) 189

P. Vilain et al., (CHARM II Collaboration), Phys. Lett. B, 313 (1993) 267

P. Vilain et al., (CHARM II Collaboration), Eur. Phys. J., C 11 (1999) 19

J. M. Conrad, M. H. Shaevitz and T. Bolton, Rev. Mod. Phys. 70 (1998) 1341

[5] N. J. Baker et al., Phys. Rev. D 43 (1991) 2765

[6] A.E. Asratyan et al., (BEBC) Z. Phys. C68 (1995) 43

[7] N. Ushida et al., (E531 collaboration), Phys.Lett.B 121 (1983) 287 and 292

[8] N. Ushida et al., (E531 collaboration), Phys.Lett.B 206 (1988) 375 and 380

[9] P. Astier et al., (NOMAD Collaboration), Phys. Lett. B 486 (2000) 35

[10] G. Ingelman., Lepto 6.1, in Proc. of Physics at HERA, Edited by W. Buchmueller, G. Ingelman, DESY, Hamburg (1992) 1366

[11] T. Sjöstrand, Computer Phys. Commun. 39 (1986) 347

T. Sjöstrand and M. Bengtsson, Computer Phys. Commun. 43 (1987) 367

T. Sjöstrand, Computer Phys. Commun. 82 (1994) 74

[12] M. Glück, E. Reya, A. Vogt, Z. Phys. C53 (1992) 127

[13] H. Plothow-Besch, Computer Phys. Commun. 75 (1993) 396

[14] A. Bodek and J. Ritchie, Phys. Rev. D 23 (1981) 1070

[15] GEANT, CERN Program Library Long Writeup W5013

[16] G. Bassompierre et al., Nucl. Inst. Meth. A 403 (1998) 363

G. Bassompierre et al., Nucl. Inst. Meth. A 411 (1998) 63

[17] Particle Data Group, The European Physical Journal C15 (2000) 1

[18] L. Lonnblad et al., JETNET 3.0 Comp. Physics Comm. 81 (1994) 185, JETNET 3.5 (1997)

[19] P.D.B. Collins and T.P. Spiller, Journal of Physics G 11 (1985) 1289

[20] C. Peterson et al., Phys. Rev. D 27 (1983) 105 